\title{
Formation of Ammonia Clusters in a Free Molecular Jet of Binary Mixtures
}

\author{
J. DA̧BEK* \\ Institute of Physics, Maria Curie-Skłodowska University, 20-031 Lublin, Poland
}
(Received July 14, 2003; revised version February 23, 2004; in final form June 24, 2004)

The formation of small ammonia clusters is investigated experimentally with a supersonic free molecular jet of $\mathrm{NH}_{3}-\mathrm{X}$ binary mixtures $(\mathrm{X}=\mathrm{Ar}$, $\mathrm{He}, \mathrm{N}_{2}$ ), with a special attention paid to the effect of second species $\mathrm{X}$ on the cluster formation. The ion currents of protonated ammonia clusters $\left(\mathrm{NH}_{3}\right)_{n} \mathrm{H}^{+}(n=2-6)$ were measured with a combined cluster source system and a double focussing sector field mass spectrometer. Experiments were made both by changing the species concentration at a constant total pressure and by changing the total pressure with a constant composition of each mixture. The $\mathrm{NH}_{3}$ mole fraction in the tested gas mixtures ranges from 10 to $100 \%$ (pure ammonia). It is observed that the formation process of ammonia clusters strongly depends on the nature of the carrier gas and its percentage in the gas mixture. The most likely explanation seems to depend on the unique properties of the free jets; the thermal anisotropy, the spatial cooling, and the mass separation effects.

PACS numbers: 36.40.-c, 47.40.Ki

\section{Introduction}

The cluster physics has its origins in the early studies of colloids, aerosols, and condensation phenomena. In recent years what has been observed is a considerable growth in the literature devoted to studies of the dynamics of formation, structure, and stability of gaseous clusters [1-4]. It is related to the wide range of physical-chemical processes in which they take part. One can enumerate here

*e-mail: dabek@tytan.umcs.lublin.pl 
a great variety of research fields ranging from solvent-solution interactions over combustion processes, atmospheric and stratospheric chemistry to biological applications. Moreover, cluster ion beams are characterised by low specific charge. This leads to a new application of accelerated cluster ion beams in science and technology, e.g., sputtering, microstructuring, thin film deposition [5-9].

An adiabatic expansion of gas is the most commonly used technique to produce the molecular beam containing clusters. In this method the respective gas is contained in the stagnation chamber at a pressure $p_{0}$ and temperature $T_{0}$. Then it expands out through a nozzle of diameter typically less than $0.1 \mathrm{~mm}$ - into an evacuated chamber. The cooled gas becomes supersaturated and condenses in the form of clusters. Cluster production continues until the expanding subject density becomes too low to promote further growth, typically a few nozzle diameters from the nozzle exit.

A number of fundamental studies of gaseous clusters (e.g. rare gases, $\mathrm{N}_{2}$, $\mathrm{CO}_{2}, \mathrm{NH}_{3}$ ) have been carried out by condensing pure gases in free jet expansion (single-component supersonic beams) but a greater variety of clusters has been generated in a supersonic expansion of binary gas mixtures. In many such experiments the subject vapour (e.g. $\mathrm{H}_{2} \mathrm{O}$, organic species, metal vapour) is mixed with an inert carrier gas and expanded through a nozzle (two-component supersonic beams). The so-called seeded molecular beams constitute a special class of two-component beams: one component (the seed gas) has a much smaller number density than the other (the carrier gas). In general, the noble carrier gas promotes the formation of clusters and the effect depends on the kind of carrier gas and its percentage in the gas mixture. To our knowledge, Milne and Green first identified this effect in 1967 [10]. They studied the influence of argon and nitrogen on the formation of $\mathrm{CO}_{2}$ dimers and found that the addition of $\mathrm{Ar}$ increases while $\mathrm{N}_{2}$ decreases cluster production.

Similar effects were observed during the historical experiment concerning the observance of fullerenes $\mathrm{C}_{60}$ and $\mathrm{C}_{70}$ in the mass carbon cluster spectra [11]. The application of helium as the carrier gas, instead of hydrogen or nitrogen, ensured the exceptionally strong dominance of the above-mentioned peaks in the mass spectrum. The level of the peaks rose with the increase in pressure of the dosed helium. Carrier gas effects occur in the research of gas heterogenic and homogenic clusters [12-14]. Some experiments show the rather specific influence of the carrier gas on the structure of the formed clusters, so-called steric effects [15-17]. For example, it has been found [16] that the type of the carrier gas affects the shape of $\mathrm{CO}_{2}-\mathrm{HCN}$ complexes (either linear or T-shaped).

Several kinds of sources have been used to obtain neutral cluster beams: Wilson and diffusion cloud chamber, adiabatic cooling in a controlled expansion Laval nozzle, and adiabatic cooling in an uncontrolled expansion free jet. Laval nozzles were first used as a molecular beam source in 1951 [18]. The seeded supersonic nozzle source is perhaps the most intense cluster beam source available [19]. 
It should be noted that other experimental techniques have frequently been used for investigation for homologous nucleation. The recent years brought many experimental and theoretical papers concerning cluster production from binary gas mixtures in conditions of condensation in thermal diffusion cloud chambers [20-22]. What follows from the analysis of these works is the lack of an explicit conclusion on the role of the carrier gas in the processes of nucleation.

The simplest and most used source in molecular beams is the orifice free jet isentropic expansion. A theoretical understanding of single-component atomic or molecular beams had reached a satisfactory level already in the 1970s [23] but molecular beams produced by expanding gas mixtures are much less understood. A majority of experiments and theoretical works devoted to the formation of clusters in a free jet of binary mixtures are performed with a very low value of the mole fraction of a condensable species, typically less than $10 \%$.

The main goal of this work is a comparison for different carrier gases how they affect the formation of ammonia microclusters in the full range of ammonia mole fraction for the investigated gas mixtures. The carrier gases have been specially selected; ammonia was seeded and antiseeded in helium (lighter carrier gas) and in nitrogen and argon (heavier carrier gases). Helium and argon, the nonstructurated noble gases, differ from each other distinctly by many physical parameters. We can enumerate for example: atomic size and mass, kinematic viscosity, thermal conductivity, etc. The formation of the clusters in free molecular jets of binary mixtures is a complex phenomenon. However, the aim of this work is to contribute to a better understanding of the processes loading to small cluster formation. The presented work is organised as follows: in Sec. 2 a brief overview of the experimental set is given, Sec. 3 presents the results and the discussion, Sec. 4 contains concluding remarks.

\section{Experimental}

In our laboratory we constructed a conventional cluster source for mass spectrometric investigations of gaseous clusters. A detailed view of the cluster beam production and the ion extraction optics of double focussing sector field mass spectrometer was presented previously [24-26]. The binary gas mixture is prepared outside the source chamber. Argon, helium, and nitrogen, at various source pressures, were employed as carrier gases. The tested beam is formed in a continuous mode by expanding a gas mixture through an axisymmetric thin-plate nozzle into a source chamber. The nozzle is made of stainless steel, the orifice $120 \mu \mathrm{m}$ deep and $40 \mu \mathrm{m}$ in diameter. The molecular beam passes downstream through a sharp-edged conical skimmer (orifice $400 \mu \mathrm{m}$, angle $60^{\circ}$ ). After some initial optimisation, the skimmer-nozzle distance is fixed at $11 \mathrm{~mm}$. The neutral ammonia clusters are ionised by electron impact with electron energies of $70 \mathrm{eV}$ and mass analysed with the use of a mass spectrometer. The measured intensities of ion currents 
are normalised in such a manner that the all plotted values are recalculated for the same sensitivity of the mass spectrometer. A majority of measurements were conducted at room temperature of the gas in the stagnation chamber. During the course of an experiment the stagnation temperature and pressure are maintained constant to within $\pm 2 \mathrm{~K}$ and $\pm 5 \mathrm{hPa}$, respectively. The background correction has been performed by subtracting the spectra measured separately with a cluster beam on and off. The plotted values represent the average from ten separate experiments. The maximum gas pressure in the stagnation chamber (up to 2 bars) is limited by the pumping speed of the applied vacuum system. Under normal operating conditions, the pressure in the ion source remains below $2 \times 10^{-5} \mathrm{hPa}$. The mass spectrometer is able to perform mass analyses in the mass range of $1-1000 \mathrm{amu}$.

\section{Results and discussion}

Ammonia is one of the most important solvent molecules able to form hydrogen bonded networks [27]. Ammonia clusters are often used as a good example for elucidating the behaviour of hydrogen-bonded complexes [28]. We have studied the influence of argon and helium as the carrier gases on the condensation process of ammonia molecules. The ion currents of $\left(\mathrm{NH}_{3}\right)_{n} \mathrm{H}^{+}$for $n=2-6$ have been measured for different compositions of $\mathrm{NH}_{3} / \mathrm{Ar}$ and $\mathrm{NH}_{3} / \mathrm{He}$ gas mixtures. The essential part of the results is shown in Figs. 1, 2, 3, and 4. Figures 1a, 2a, 3a, 4a refer to $\mathrm{NH}_{3} / \mathrm{Ar}$ and Figs. 1b, 2b, 3b, $4 \mathrm{~b}$ to $\mathrm{NH}_{3} / \mathrm{He}$ mixtures. The only difference in experimental conditions of (a) and (b) measurements is the nature of the carrier gas. The other parameters of the tested mixtures, i.e. stagnation pressure $\left(p_{0}\right)$ and stagnation temperature $\left(T_{0}\right)$, were the same. Also the crucial parameters in the ion source operation, i.e. the intensity and energy of the electron beam were the same.

Even a casual inspection of Fig. 1a and b allows one to notice a distinct difference in the pattern of plots. As the mole fraction of ammonia in the $\mathrm{NH}_{3} / \mathrm{Ar}$ mixture decreases the ion current of $\left(\mathrm{NH}_{3}\right)_{2} \mathrm{H}^{+}$decreases monotonically (see Fig. 1a). On the contrary, if the mole fraction of ammonia in the $\mathrm{NH}_{3} / \mathrm{He}$ mixture decreases, the measured $\left(\mathrm{NH}_{3}\right)_{2} \mathrm{H}^{+}$intensity increases and then rapidly decreases after reaching a maximum (see Fig. 1b). Nearly the same pattern, but for protonated ammonia trimers is visible in Fig. 2, especially for the $\mathrm{NH}_{3} / \mathrm{He}$ mixtures. The formation of protonated ammonia dimers, trimers, and tetramers below room temperature $\left(T_{0}=250 \mathrm{~K}\right)$ as a function of abundance of ammonia in examined mixtures is shown in Fig. 3. As the mole fraction of ammonia in the $\mathrm{NH}_{3} / \mathrm{Ar}$ mixture decreases the ion signal of $\left(\mathrm{NH}_{3}\right)_{2} \mathrm{H}^{+}$and $\left(\mathrm{NH}_{3}\right)_{3} \mathrm{H}^{+}$decrease drastically (Fig. 3a). In the case of the $\mathrm{NH}_{3} / \mathrm{He}$ mixtures (Fig. 3b), the influence of helium carrier gas is the most visible. A maximum of $\left(\mathrm{NH}_{3}\right)_{2} \mathrm{H}^{+}$intensity is obtained at about $30 \%$ of ammonia in the gas source. The formation of ammonia dimers and 

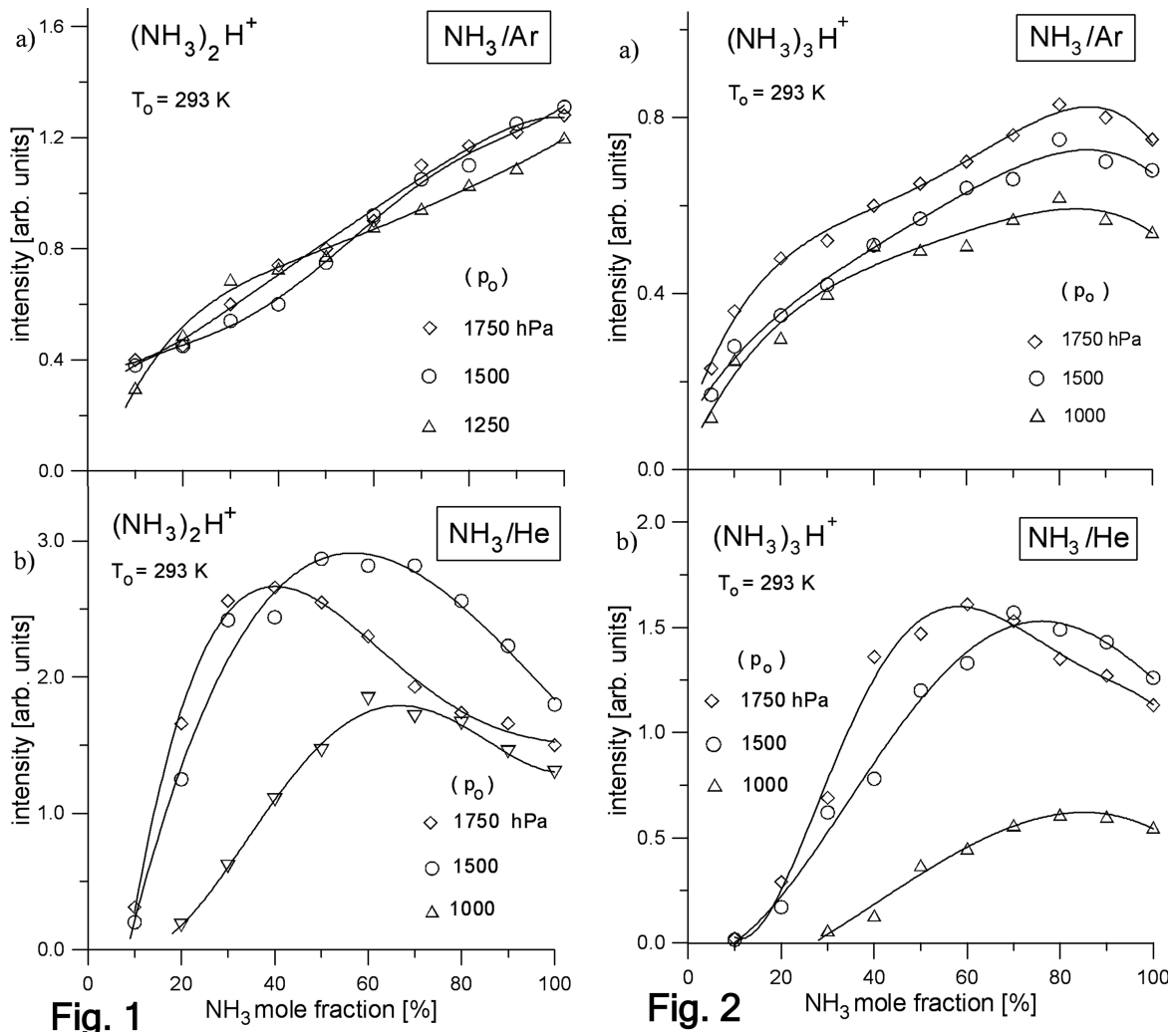

Fig. 1. Concentration of protonated ammonia dimers as a function of ammonia mole fraction in the gas mixture at stagnation temperature $T_{0}=293 \mathrm{~K}$ and at three values of stagnation pressure for (a) $\mathrm{NH}_{3} / \mathrm{Ar}$ mixture and (b) $\mathrm{NH}_{3} / \mathrm{He}$ mixture.

Fig. 2. Concentration of protonated ammonia trimers as a function of ammonia mole fraction in the gas mixture at stagnation temperature $T_{0}=293 \mathrm{~K}$ and at three values of stagnation pressure for (a) $\mathrm{NH}_{3} / \mathrm{Ar}$ mixture and (b) $\mathrm{NH}_{3} / \mathrm{He}$ mixture.

trimers as a function of the total pressure for the neat ammonia and two values of the ammonia mole fraction ( $80 \%$ and $50 \%$ ) are shown in Fig. 4 and 5, respectively. In general, the presented plots are compatible to the results presented in Figs. 1, 2. In many experiments connected to the study of the condensation processes nitrogen was applied as a carrier gas. In this work the effect of nitrogen on formation of ammonia clusters was examined too (Fig. 6). In the case of $\mathrm{NH}_{3} / \mathrm{N}_{2}$ and $\mathrm{NH}_{3} /$ Ar mixtures the mass fractionation mechanism may negatively affects the $\mathrm{NH}_{3}$ cluster signal. The heavier $\mathrm{N}_{2}$ molecules or Ar atoms are focussed to the centreline of the beam while the $\mathrm{NH}_{3}$ molecules are segregated to the edge of the beam. For the $\mathrm{NH}_{3} / \mathrm{He}$ mixture the light carrier gas concentrates ammonia molecules on the axis of flow. This angular mass distribution directly results from an inertial effect, which take place within the supersonic molecular beams. The 


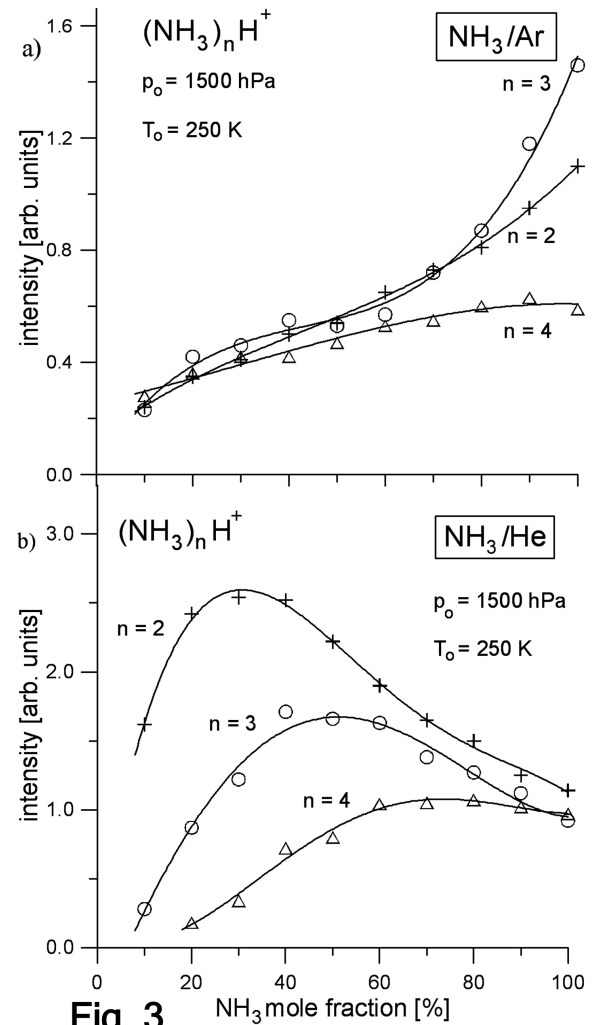

Fig. 3

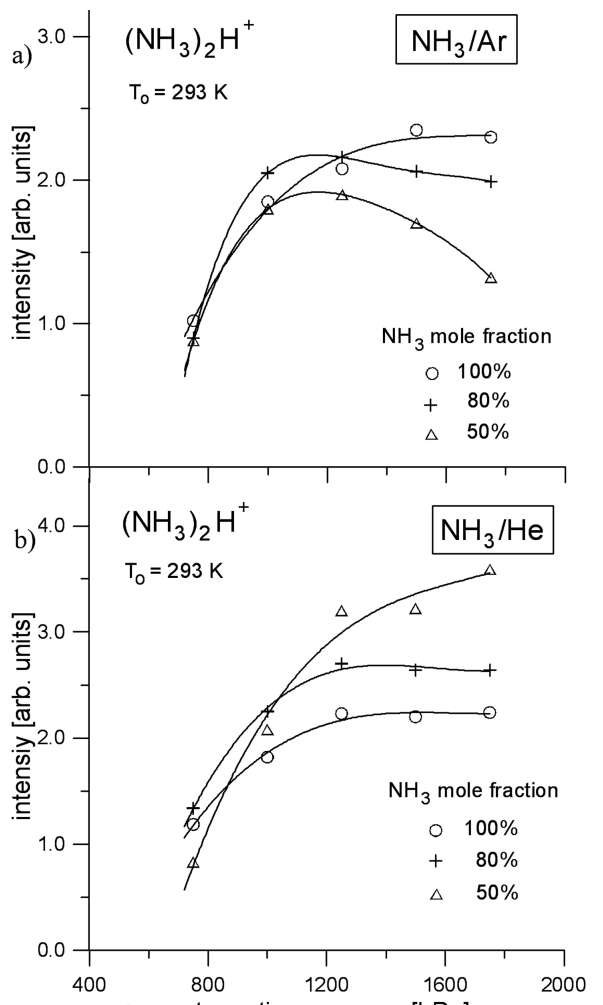

Fig. 4

Fig. 3. Concentration of protonated ammonia dimers, trimers, and tetramers as a function of ammonia mole fraction in the gas mixture at stagnation temperature $T_{0}=250 \mathrm{~K}$ and at stagnation pressure $p_{0}=1500 \mathrm{hPa}$ for (a) $\mathrm{NH}_{3} / \mathrm{Ar}$ mixture and (b) $\mathrm{NH}_{3} / \mathrm{He}$ mixture.

Fig. 4. Formation of ammonia dimers as a function of stagnation pressure at stagnation temperature $T_{0}=293 \mathrm{~K}$ and at three values of ammonia mole fraction for (a) $\mathrm{NH}_{3} / \mathrm{Ar}$ mixture and (b) $\mathrm{NH}_{3} / \mathrm{He}$ mixture.

different role of an inert gas in the formation of protonated ammonia dimers for the three gas mixtures: $\mathrm{NH}_{3} / \mathrm{Ar}, \mathrm{NH}_{3} / \mathrm{N}_{2}$, and $\mathrm{NH}_{3} / \mathrm{He}$ are shown in Fig. 7 . From the compared gases, helium with its high thermal conductivity is best effective as a carrier gas for the productions of clusters. The formation of the higher ammonia clusters $\left(n=5\right.$ and $n=6$ ) in free molecular expansion of $\mathrm{NH}_{3} / \mathrm{Ar}$ mixtures for two values of the stagnation gas temperature is shown in Fig. 8a and b. The obtained plots are somewhat unusual in the shape. The intensities of $\left(\mathrm{NH}_{3}\right)_{5} \mathrm{H}^{+}$ and $\left(\mathrm{NH}_{3}\right)_{6} \mathrm{H}^{+}$ion currents obtained at $T_{0}=293 \mathrm{~K}$ stagnation temperature show the distinct maximum for $50 \%$ and $30 \%$ ammonia mole fraction.

Similar measurements but for protonated water clusters were performed by Inoue and Kotake [14]. They investigated experimentally the water clusters with 


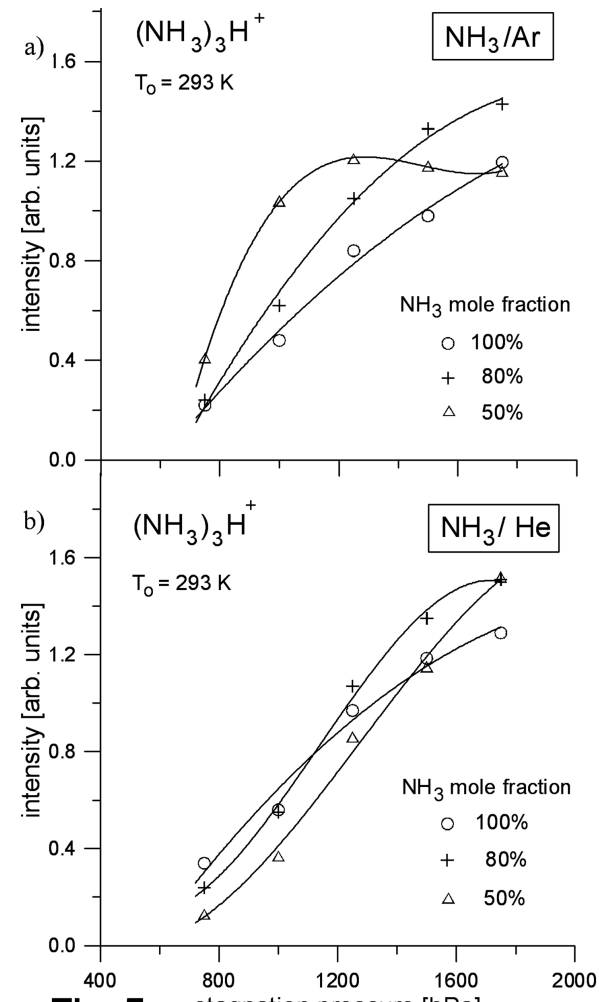

Fig. 5

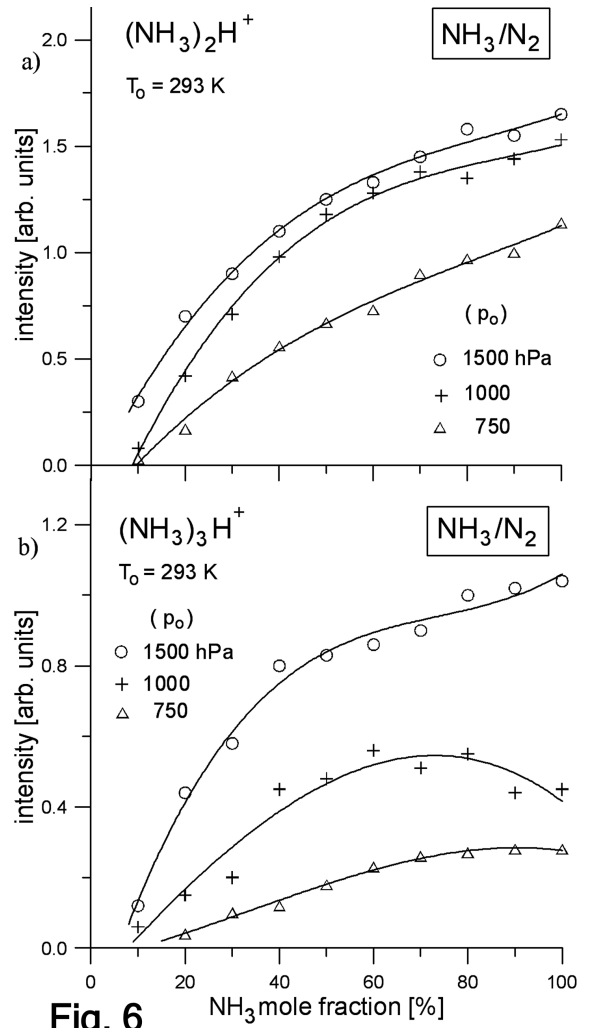

Fig. 6

Fig. 5. Formation of ammonia trimers as a function of stagnation pressure at stagnation temperature $T_{0}=293 \mathrm{~K}$ and at three values of ammonia mole fraction for (a) $\mathrm{NH}_{3} / \mathrm{Ar}$ mixture and (b) $\mathrm{NH}_{3} / \mathrm{He}$ mixture.

Fig. 6. Formation of protonated ammonia dimers (a) and protonated ammonia trimers (b) as a function of ammonia mole fraction in $\mathrm{NH}_{3} / \mathrm{N}_{2}$ mixture.

a supersonic free molecular jet of water vapour mixtures of $\mathrm{Ar}, \mathrm{Xe}, \mathrm{N}_{2}, \mathrm{CO}_{2}$, and CO. In this work the dimer concentration was little affected by the applied carrier gases. The concentrations of larger water clusters showed a maximum at the $\mathrm{H}_{2} \mathrm{O}$ mole fraction of about $65 \%$ for Ar, Xe mixtures and of about $85 \%$ for $\mathrm{CO}, \mathrm{CO}_{2}$ mixtures. In $\mathrm{H}_{2} \mathrm{O} / \mathrm{N}_{2}$ system the intensity of water clusters changed monotonically without any marked evidence of the maximum [14].

At the beginning of the discussion of the presented results it is necessary to point out that the ion intensities of neutral clusters after ionisation are not simply correlated with their neutral precursors. Thus, the observed ion mass spectra may differ from the original neutral cluster distribution. In general, a cluster ion mass spectrum produced by electron impact shows the parent ions and ionic fragments of a larger cluster that are produced in the source region, as well. In the case of a low condensed molecular beam, produced under moderate expansion, the formation of 

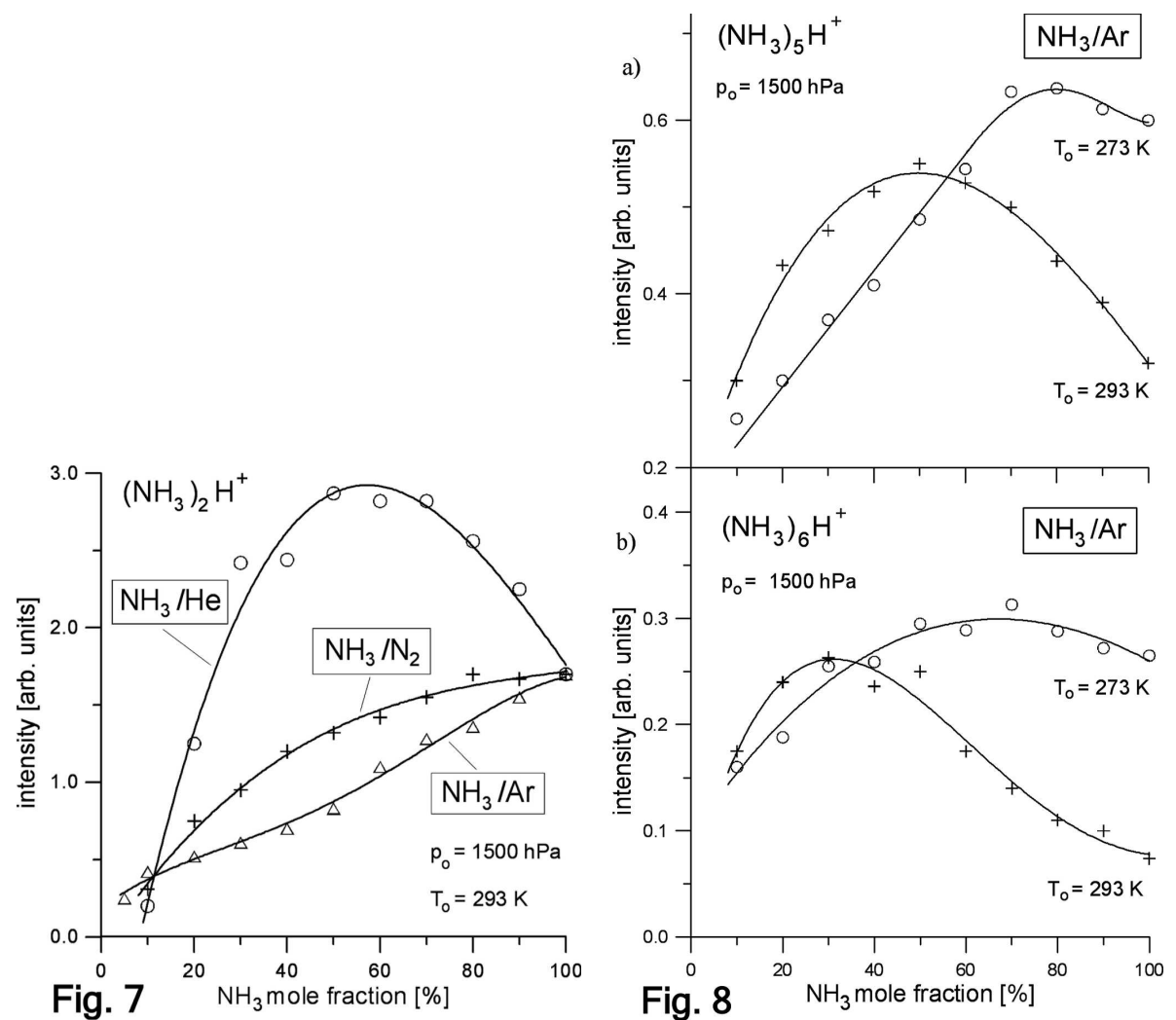

Fig. 7. Comparison of protonated ammonia dimers formation as a function of ammonia mole fraction for the three gas mixtures at stagnation temperature $T_{0}=293 \mathrm{~K}$ and stagnation pressure $p_{0}=1500 \mathrm{hPa}$.

Fig. 8. Formation of protonated ammonia pentamers (a) and hexamers (b) as a function of ammonia mole fraction in $\mathrm{NH}_{3} / \mathrm{Ar}$ mixture at stagnation pressure $p_{0}=1500 \mathrm{hPa}$ and at two values of stagnation temperature.

dimers as a results of the fragmentation of larger clusters can be neglected [2, 29]. Moreover, during the course of these experiments, the crucial parameters of the ion source operation were kept constant. Thus, we have a reason to believe that the observed changes in the cluster ion intensities are mainly caused by a change of the composition of expanded beam.

A change in the composition of the expanded beam affects the formation process of clusters in various ways. Firstly, as the ammonia mole fraction in the mixture decreases, the amount of condensing species is decreased too (dilution effect). Secondly, the change in the specific heat ratio of a binary mixture leads to a change of the thermodynamic factors of the beam (so-called gamma effect) [14]. Thirdly, the change in the dispersion of translational velocity due to the mass difference in the gas mixture affects the kinetic parameters of the expanded beam 
(i.e. particle velocity, frequency of collisions, reactive collision cross-section). It should be pointed out that the gas expands radially and axially, accelerating axially to a supersonic velocity. Finally, the participation of a third body (ammonia molecule or an atom of inert gas) in the particle collisions affects the exchange of the internal energy between an excited cluster and the surrounding molecules. A monoatomic carrier gas used with molecular vapours, e.g., $\mathrm{He} / \mathrm{NH}_{3}$, serves as a medium to cool the growing clusters and to absorb the heat of condensation. Among the gases under investigation, helium with its high thermal conductivity is best suited as carrier gas for the formation of clusters (see Table I).

TABLE I

A summary of the properties of the carrier gases used in this study [30].

\begin{tabular}{l|l|c|c}
\hline \hline \multicolumn{1}{c|}{ Parameter } & \multicolumn{3}{c}{ Gas } \\
\cline { 2 - 4 } & Argon & Helium & Nitrogen \\
\hline density $\left(\mathrm{kg} \mathrm{m}^{-1}\right)$ & 1.7839 & 0.1785 & 1.1383 \\
dynamic viscosity $\left(10^{6} \mathrm{~N} \mathrm{~s} \mathrm{~m}^{-2}\right)$ & 2.270 & 1.9788 & 1.7855 \\
kinematic viscosity $\left(10^{5} \mathrm{~m}^{2} \mathrm{~s}^{-1}\right)$ & 1.2725 & 11.0857 & 1.5686 \\
thermal conductivity $\left(10^{2} \mathrm{~W}^{-1} \mathrm{~K}^{-1} \mathrm{~m}^{-1}\right)$ & 1.788 & 15.336 & 2.6052
\end{tabular}

In a typical free jet, the internal energy of the molecules in the source is transferred into directional kinetic energy of the flow during the supersonic expansion. The highest values of the cooling and rarefaction rate are obtained near the nozzle. At the distance of only 10 nozzle diameters downstream from nozzle exit, the temperature of a monoatomic gas is only $1 \%$ or $2 \%$ of its source value [31]. Because the flow velocities are a few times $10^{4} \mathrm{~cm} / \mathrm{s}$, the cooling rate of the gas is well over $10^{8} \mathrm{~K} / \mathrm{s}$.

During the supersonic expansion of the beam of polyatomic molecules the rotational, vibrational, and translational degrees of freedom are significantly cooled. So, the expanded molecular beam is a thermally anisotropic and nonequilibrium medium. It is a common practice to describe the expanded beam by two temperature terms. One of them describing the random motion along the direction of the streamline and another the motion perpendicular to this axis [4, 23]. In general, the order of temperatures is $T_{\text {vib }}>T_{\text {rot }} \gg T_{\text {trans } \|}>T_{\text {trans } \perp}$ [4]. Supersonic flows are characterised by the Mach number $M=v / c$, where $v$ is the mean flow velocity and $c$ is the local speed of sound. Note that the increase in $M$ is mainly caused by the decrease in $c$ associated with the gas cooling. The velocity of the sound decreases as $T^{1 / 2}$. For an ideal gas the translational temperature at a given point $(x)$ in the beam is related to the stagnation gas temperature $T_{0}$ by $[2,23]$

$$
\left.T(x)=T_{0}\{1+[(\gamma-1) / 2)] M^{2}(x)\right\}^{-1} .
$$

As the expansion proceeds, Mach number increases until it approaches a terminal 
value $M_{\mathrm{t}}$. For the terminal Mach number $M_{\mathrm{t}}$ Anderson and Fenn [31] found the approximate value

$$
M_{\mathrm{t}}=c^{\prime}\left(p_{0} d\right)^{0.4},
$$

where $p_{0}$ is the stagnation pressure (bar), $d$ is the nozzle diameter $(\mathrm{cm})$ and $c^{\prime}$ is the constant which for argon takes the value 135. For parameters typical of the experiments of this work $\left(p_{0}=2\right.$ bar and $d=0.004 \mathrm{~cm}$ ) one arrives at $M_{\mathrm{t}}=19.5$. The combination of Eqs. (1) and (2) leads to the terminal beam temperature of $2 \mathrm{~K}$. In the initial collision-dominated regime the expansion is isentropic with flow velocity increasing to its limit $w_{\max }[32,33]$. The maximum flow velocity is about 1.5 times larger than the average thermal velocity in the source [34]

$$
w_{\max }=1.581\left(2 k T_{0} / m\right)^{1 / 2}=204\left(T_{0} / m\right)^{1 / 2}[\mathrm{~m} / \mathrm{s}] \text { if } T_{0}[\mathrm{~K}] \text { and } m[\mathrm{amu}] \text {. }
$$

In a mixture of a heavy gas seeded in a light gas, the difference in the masses and average thermal velocities of the gases in the stagnation chamber translates into a difference in axially directed velocities as the expansion develops from random motion to a uniform bulk velocity. The so-called velocity slip occurs in the early stages of expansion and is directed mainly along the expansion axis $[17,35,36]$. A simple way to vary the velocity slip and average collision partner is to change the relative concentration of seed and carrier gases for the expanded mixture, for example $\mathrm{NH}_{3} / \mathrm{He}$. For higher concentrations of $\mathrm{NH}_{3}$, a greater proportion of the binary collisions are between two molecules of ammonia. The value of velocity slip parameter $(V S P)$ increases with decreasing $\mathrm{NH}_{3}$ concentration, achieving the maximum value for low concentration, where the $\mathrm{NH}_{3}-\mathrm{He}$ collisions dominate. The theoretical description for the terminal velocity slip of a binary expansion is given by equation

$$
\Delta w_{\max } / w_{\max } \cong 1 / V S P,
$$

where $w_{\max }$ is the terminal, isentropic velocity for a binary expansion of gases and $\Delta w_{\max }$ is the difference in terminal velocities for the two components of the expanded beam [17].

There are many reasons for using a supersonic expansion of binary gas mixtures. Menzel and co-workers [37] scattered the ammonia clusters off a $\operatorname{LiF}(100)$ surface. The clusters were produced in an adiabatic expansion using different gas mixtures in order to vary the cluster velocity. In their experiments slow clusters were produced in the expansion of a 1:1 gas mixture of $\mathrm{NH}_{3}$ and $\mathrm{Kr}\left(E_{\text {kin }}=58 \mathrm{meV}\right.$ per monomer). Clusters at medium velocity were formed in the expansion of pure ammonia $\left(E_{\mathrm{kin}}=176 \mathrm{meV}\right.$ per monomer $)$. Finally, fast clusters were obtained by the expansion of a 1:3 gas mixture of $\mathrm{NH}_{3}$ and $\mathrm{He}\left(E_{\text {kin }}=285 \mathrm{meV}\right.$ per monomer) [37]. Free jet expansion of binary atomic mixtures was studied theoretically by Mazely et al. [38]. They used a method of moments solutions of the Boltzman equation to describe flow and thermal relaxation for the free jets. They stated that two phenomena are seen to play major roles in mixture flows: velocity 
TABLE II

Expansion conditions for ammonia cluster formation.

\begin{tabular}{|c|c|c|c|c|c|c|}
\hline $\begin{array}{l}\text { Subject }+ \\
\text { carrier gas }\end{array}$ & $\begin{array}{c}\text { Subject mole } \\
\text { fraction }\end{array}$ & $\begin{array}{c}M_{\mathrm{m}}^{a} \\
(\mathrm{amu})\end{array}$ & $f_{\mathrm{m}}^{b}$ & $\begin{array}{l}w_{\max }^{c} \\
(\mathrm{~m} / \mathrm{s})\end{array}$ & $1 / V S P^{d}$ & $\begin{array}{c}T_{b}(\mathrm{~K})^{e} \\
\text { (for } M=5 \text { ) }\end{array}$ \\
\hline \multirow{9}{*}{$\mathrm{NH}_{3}+\mathrm{Ar}$} & 0.1 & 37.7 & 3.3 & 585.4 & -0.887 & 34.2 \\
\hline & 0.2 & 35.4 & 3.6 & 621.0 & -0.835 & 36.9 \\
\hline & 0.3 & 33.1 & 3.9 & 659.2 & -0.787 & 39.5 \\
\hline & 0.4 & 30.8 & 4.2 & 700.5 & -0.741 & 42.1 \\
\hline & 0.5 & 28.5 & 4.5 & 745.7 & -0.696 & 44.7 \\
\hline & 0.6 & 26.2 & 4.8 & 795.4 & -0.653 & 47.2 \\
\hline & 0.7 & 23.9 & 5.1 & 851.0 & -0.610 & 49.6 \\
\hline & 0.8 & 21.6 & 5.4 & 913.9 & -0.568 & 52.0 \\
\hline & 0.9 & 19.3 & 5.7 & 986.6 & -0.526 & 54.4 \\
\hline \multirow[t]{9}{*}{$\mathrm{NH}_{3}+\mathrm{He}$} & 0.1 & 5.3 & 3.3 & 1561.4 & 0.432 & 34.2 \\
\hline & 0.2 & 6.6 & 3.6 & 1438.2 & 0.469 & 36.9 \\
\hline & 0.3 & 7.9 & 3.9 & 1349.3 & 0.500 & 39.5 \\
\hline & 0.4 & 9.2 & 4.2 & 1281.8 & 0.526 & 42.1 \\
\hline & 0.5 & 10.5 & 4.5 & 1228.5 & 0.549 & 44.7 \\
\hline & 0.6 & 11.8 & 4.8 & 1185.3 & 0.569 & 47.2 \\
\hline & 0.7 & 13.1 & 5.1 & 1149.5 & 0.587 & 49.6 \\
\hline & 0.8 & 14.4 & 5.4 & 1119.3 & 0.603 & 52.0 \\
\hline & 0.9 & 15.7 & 5.7 & 1093.5 & 0.620 & 54.4 \\
\hline \multirow[t]{9}{*}{$\mathrm{NH}_{3}+\mathrm{N}_{2}$} & 0.1 & 26.9 & 5.1 & 802.2 & -0.362 & 49.6 \\
\hline & 0.2 & 25.8 & 5.2 & 824.8 & -0.352 & 50.4 \\
\hline & 0.3 & 24.7 & 5.3 & 848.8 & -0.342 & 51.2 \\
\hline & 0.4 & 23.6 & 5.4 & 874.3 & -0.332 & 52.0 \\
\hline & 0.5 & 22.5 & 5.5 & 901.5 & -0.322 & 52.9 \\
\hline & 0.6 & 21.4 & 5.6 & 930.5 & -0.312 & 53.6 \\
\hline & 0.7 & 20.3 & 5.7 & 961.6 & -0.302 & 54.4 \\
\hline & 0.8 & 19.2 & 5.8 & 995.2 & -0.292 & 55.2 \\
\hline & 0.9 & 18.1 & 5.9 & 1031.5 & -0.281 & 56.0 \\
\hline neat $\mathrm{NH}_{3}$ & 1.0 & 17.0 & 6.0 & 1071.1 & & 56.7 \\
\hline
\end{tabular}

slip and temperature slip. Moreover, as implies in the calculations, the velocity slip maximises near a mole fraction of $50 \%$ (for 1:1 mixtures). In other works $[39,40]$ the transport coefficients such as the viscosity, thermal conductivity for gaseous mixtures were measured. The dependence of these coefficients on the mixture composition was measured as a function of the mole fraction. Three different gases $\left(\mathrm{Ar}, \mathrm{Ne}\right.$, and $\mathrm{N}_{2}$ ) were studied in conjunction with helium. The velocity 
and viscosity slip coefficients as a function of the mixture composition showed a maximum. These conclusions correspond to the maximum of ammonia dimer formation obtained in the presented work (see Figs. 1b, 2b, and 3b). It should be noted that a maximum probability of the binary collisions between carrier atoms and ammonia molecules occurs for 1:1 gas mixtures.

In binary gas mixtures different species have different collision cross-sections, masses, intermolecular potentials, polarities, structures, and energies. If a binary gas mixture consists of an inert carrier gas (index g) and a condensing vapour (index v), we can calculate an average value of the mixture mass $\left(m_{\mathrm{m}}\right)$ and an average value of the number of degrees of freedom $\left(f_{\mathrm{m}}\right)[30,31]$

$$
m_{\mathrm{m}}=X_{\mathrm{v}} m_{\mathrm{v}}+\left(1-X_{\mathrm{v}}\right) m_{\mathrm{g}}, \quad f_{\mathrm{m}}=X_{\mathrm{v}} f_{\mathrm{v}}+\left(1-X_{\mathrm{v}}\right) f_{\mathrm{g}},
$$

where $X_{\mathrm{v}}$ - mole fraction of the vapour. Since the specific heat ratio of Ar and He takes the same value, 1.67, then the difference in the formation of ammonia clusters between $\mathrm{NH}_{3} / \mathrm{Ar}$ and $\mathrm{NH}_{3} / \mathrm{He}$ binary mixtures are caused mainly by the kinetic parameters of expanded beam. Note that the increase in argon mole fraction in the $\mathrm{NH}_{3} / \mathrm{Ar}$ mixture decreases the velocities of ammonia dimmers. This reflects the increasing mean atomic mass of the $\mathrm{NH}_{3} / \mathrm{Ar}$ mixture. For the $\mathrm{NH}_{3} / \mathrm{He}$ mixture just the opposite is true. Some expansion parameters for ammonia cluster formation, obtained from Eqs. (1), (3), (4), and (5) are shown on Table II. The beam parameters inserted in this table are calculated on the ground of a simple one-dimensional model of the gas expansion. The real structure of the molecular beam of the gas mixture is considerably more complex. One can enumerate a number of the gas dynamic parameters that depend on the mixture composition, for example: the Reynold number, viscosity, thermal conductivity, variety of collision pairs, and cross-section of internal relaxation of the molecular species. The problem of the intrajet collisions can arise, particularly when the expanded beam consists of a polar species.

\section{Conclusions}

We have presented a comparative study of the formation of small ammonia clusters in a supersonic expansion of binary gas mixtures as a function of ammonia mole fraction. In the examined gas mixtures $\mathrm{NH}_{3}-\mathrm{X}$, where $\mathrm{X}=\mathrm{He}$, Ar or $\mathrm{N}_{2}$ the $\mathrm{NH}_{3}$ mole fraction was changed from 0.1 to 1 . We have found that in a selected regime of the experimental conditions $\left(p_{0}=1000-1750 \mathrm{hPa}\right.$ and $\left.T_{0}=250-293 \mathrm{~K}\right)$ the formation of ammonia microclusters strongly depends on the nature of the carrier gas and its percentage in the gas mixture.

The commonly applied explanation of the carrier gas effect on the cluster formation during the expansion of the gas mixtures underlines only the cooling effect. The inert gas acts as thermostat to cool the growing clusters and to absorb the heat of condensation [5]. Such explanation is especially satisfactory for the diluted gas mixtures (seeded beams). The second role of helium as a carrier gas consist in 
a mass fractionation mechanism, which greatly enhances the cluster signal. The expanded beam of a gaseous mixture is a nonequilibrium medium. A direct consequence of the nonequilibrium conditions are the velocity and temperature slip phenomena observed in the free jets. Moreover, the distribution of particle size within a jet expanded from a nozzle is not uniform. The heavier species of the gas mixture (for example argon as a carrier gas in the $\mathrm{NH}_{3} / \mathrm{Ar}$ mixture) is focused to the centreline of the expanded beam while the lighter species (for example helium as a carrier gas in the $\mathrm{NH}_{3} / \mathrm{He}$ mixture) is segregated to the edge of the beam [31, 41, 42]. Taking into account the linear structure of ammonia dimers one can classify these clusters as a "ball and ball"-type clusters. So, we also supposed that the rotational angular momentum of ammonia dimers is aligned substantially in the co-expansion with helium. Similar steric effects induced in molecular collisions were observed for iodine and carbon dioxide molecules seeded in supersonic beams of light carrier gases [15, 17]. It is commonly assumed that no detailed quantitative theories of the cluster formation in the supersonic expansion of binary gas mixtures exist. For this reason, the systematic experiments regarding the beam of complex gas mixtures are of general value.

\section{References}

[1] T.D. Märk, Int. J. Mass Spectrom. Ion Processes 79, 1 (1987).

[2] H. Haberland, in: Clusters of Atoms and Molecules I, Ed. H. Haberland, Vol. 52, Springer-Verlag, Berlin 1994, p. 205.

[3] A.W. Castelman Jr., K.H. Bowen Jr., J. Phys. Chem. 100, 12911 (1996).

[4] K.M. Weitzel, J. Mähnert, Int. J. Mass Spectrom. 214, 17 (2002).

[5] O.F. Hagena, Rev. Sci. Instrum. 63, 2374 (1992).

[6] H. Haberland, M. Karrais, M. Mall, Y. Thurner, J. Vac. Sci. Technol. A 10, 3266 (1992).

[7] B.A. Ferguson, C.B. Mullins, J. Cryst. Growth 178, 134 (1997).

[8] C. Binns, Surf. Sci. Rep. 44, 1 (2001).

[9] I. Yamada, J. Matsuo, N. Toyoda, A. Kirkpatrick, Mater. Sci. Eng. R 34, 2 (2001).

[10] T.A. Milne, F.T. Greene, J. Chem. Phys. 47, 4095 (1967).

[11] H.W. Kroto, J.R. Heath, S.C. O'Brien, R.F. Curl, R.E. Smaley, Nature 318, 162 (1985).

[12] M. Yamashita, T. Sano, S. Kotake, J. Chem. Phys. 75, 5355 (1981).

[13] A. Ding, J.H. Futrel, R.A. Cassidy, L. Cordis, J. Hesslich, Surf. Sci. 156, 282 (1985).

[14] T. Inoue, S. Kotake, J. Chem. Phys. 91, 162 (1989).

[15] D.P. Pullman, B. Friedrich, D.R. Herschbach, J. Chem. Phys. 92, 3224 (1990).

[16] T.D. Klots, R.S. Ruoff, H.S. Gutowsky, J. Chem. Phys. 90, 4216 (1988). 
[17] M.J. Weida, D.J. Nesbitt, J. Chem. Phys. 100, 6372 (1994).

[18] G.D. Stein, Surf. Sci. 156, 44 (1985).

[19] W.A. De Heer, Rev. Mod. Phys. 65, 611 (1993).

[20] D. Kashchiev, J. Chem. Phys. 104, 8671 (1996).

[21] D. Kane, S.P. Fisenko, M. Rusyniak, M.S. El-Shall, J. Chem. Phys. 111, 8496 (1999).

[22] K.J. Oh, X.C. Zeng, J. Chem. Phys. 114, 2681 (2001).

[23] S. DePaul, D. Pullman, B. Friedrich, J. Phys. Chem. 97, 2167 (1992).

[24] J. Dąbek, L. Michalak, Nukleonika 44, 293 (1999).

[25] A. Pelc, L. Michalak, Vacuum 52, 261 (1999).

[26] J. Dạbek, L. Michalak, Vacuum 61, 555 (2001).

[27] K.A. Walker, A.R.W. Mckellar, Mol. Phys. 99, 1397 (2001).

[28] E.M. Snyder, J. Purnell, S. Wei, S.A. Buzza, A. W. Castelman Jr., Chem. Phys. 207, 353 (1996).

[29] C. Winkler, Chem. Phys. Lett. 242, 39 (1995).

[30] R.H. Perry, D. Green, Perry's Chemical Engineer's Handbook, 6th ed., McGraw-Hill, New York 1984, Ch. 5.

[31] J.B. Fenn, Int. J. Mass Spectrom. 200, 459 (2000).

[32] O.F. Hagena, Surf. Sci. 106, 101 (1981).

[33] G. Callies, H. Schittenhelm, P. Berger, H. Hügel, Appl. Surf. Sci. 127-129, 134 (1998).

[34] V.J. Herrero, I. Tanarro, Vacuum 52, 3 (1999).

[35] L.-Q. Xia, M.E. Jones. N. Maity, J.R. Engstrom, J. Vac. Sci. Technol. A 13, 2651 (1995).

[36] M. Rosemeyer, R. Schäfer, J.A. Becker, Chem. Phys. Lett. 339, 323 (2001).

[37] C. Menzel, R. Baumfalk, H. Zacharias, Chem. Phys. 239, 287 (1998).

[38] T.L. Mazely, G.H. Roehrig, M.A. Smith, J. Chem. Phys. 103, 8638 (1995).

[39] I.N. Ivchenko, S.K. Loyalka, R.V. Tompson, J. Vac. Sci. Technol. A 15, 2375 (1997).

[40] J.A. Bentz, R.V. Tompson, S.K. Loyalka, J. Vac. Sci. Technol. A 17, 235 (1999).

[41] A.J. McGinnis, D. Thomson, R.F. Davis, E. Chen, A. Michel, H.H. Lamb, Surf. Sci. 494, 28 (2001).

[42] L.S. Bartell, J. Phys. Chem. 94, 5102 (1990). 